\title{
Recommendations for the Long-Term Treatment of Psoriasis with Infliximab: A Dermatology Expert Group Consensus
}

\author{
Kristian Reich $^{a} \quad$ Christopher Griffiths $^{d} \quad$ Jonathan Barker ${ }^{e} \quad$ Sergio Chimenti ${ }^{f}$ \\ Esteban Daudén $^{\mathrm{h}}$ Alberto Giannettig ${ }^{\mathrm{g}}$ Robert Gniadecki ${ }^{\mathrm{i}} \quad$ Andreas Katsambas $^{\mathrm{j}}$ \\ Richard Langley $^{k} \quad$ Ulrich Mrowietz ${ }^{b} \quad$ Alexandra Ogilvie $^{c}$ Jean-Paul Ortonnel \\ Norbert Reider ${ }^{\mathrm{m}}$ Jean-Hilaire Saurat ${ }^{\mathrm{n}}$ \\ a Department of Dermatology, Dermatologikum Hamburg, Hamburg, ${ }^{b}$ Psoriasis Center at the Department of \\ Dermatology, University Clinic of Schleswig-Holstein, Campus Kiel, Kiel, and ${ }^{\mathrm{C}}$ Hautklinik des Universitätsklinikums \\ Erlangen, Erlangen, Germany; ${ }^{\mathrm{d}}$ Dermatology Centre, Hope Hospital, University of Manchester, Manchester, and \\ eSt. John's Institute of Dermatology, St. Thomas' Hospital, London, UK; ${ }^{\mathrm{f}}$ Department of Dermatology, University

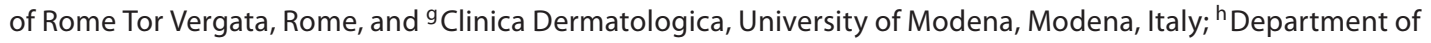 \\ Dermatology, Hospital Universitario de la Princesa, Madrid, Spain; 'Bispebjerg Hospital, København, Denmark; \\ jDermatology Clinic, Athens University, Athens, Greece; kGB Dalhousie University, Halifax, N.S., Canada; \\ 'Department of Dermatology, Hôpital I'Archet 2, Nice, France; 'm Department of Dermatology, Medical University \\ of Innsbruck, Innsbruck, Austria; n'Department of Dermatology, University of Geneva, Geneva, Switzerland
}

\section{Key Words}

Psoriasis, long-term treatment • Biological agents • Infliximab · Consensus

\begin{abstract}
Background/Aims: Infliximab has been approved for the treatment of chronic plaque psoriasis for only a few years. As physicians gain confidence in initiating and maintaining this therapy, guidance on the management of patients beyond several months or years is needed. To date, there is little or no information about the long-term management in clinical trials or guidelines. Methods: Here we report on the key aspects related to the use of infliximab for the treatment of psoriasis. The data presented here were derived using a modified Delphi survey to obtain a consensus opinion of 11 dermatologists from Europe and Canada experienced in long-term therapy with infliximab. Results/Conclusion: The Delphi participants reviewed several important topics relat-
\end{abstract}

\section{KARGER \\ Fax +41613061234 \\ E-Mail karger@karger.ch}

www.karger.com

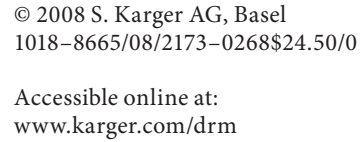

ed to biological therapy and infliximab. This paper is not intended to provide a recommendation on all practical aspects related to biological therapy; it has rather been written to provide useful and practical information on the 'best practice' use of infliximab.

Copyright $\odot 2008$ S. Karger AG, Basel

\section{Introduction}

Infliximab, a chimeric monoclonal antibody against human tumour necrosis factor $\alpha$, has been successfully used in the treatment of a number of auto-immune disorders, including ulcerative colitis, adult and paediatric Crohn's disease (CD), ankylosing spondylitis, rheumatoid arthritis, psoriasis and psoriatic arthritis (PsA). Worldwide, nearly 1 million patients have been treated with infliximab, and its benefit:risk profile is therefore well characterised. Infliximab has been approved in Eu- 


\author{
Steering group \\ Qualifications \\ - Possess extensive experience in using biological \\ therapy \\ - Involved in numerous clinical trials investigating \\ biological therapy in plaque psoriasis \\ Responsibilities \\ - Outline topics for survey \\ - Provide input on survey design and methodology \\ - Review survey data prior to release to participants \\ - Present data to expert panel
}

\section{Expert panel}

Qualifications

- Significant experience using infliximab for the long-term treatment of patients with moderate to severe psoriasis (clinical trials and clinical practice)

- Possess a combined 66 years of experience using biological agents (median 6 years; range $3-10$ years) and a combined 51 years of familiarity with prescribing infliximab (median 4 years; range $2-8$ years) Responsibilities

- Participate in 2 rounds of an anonymous questionnaire addressing the use of biological agents

- Attend an on-site meeting to discuss survey results and develop additional questions on topics of interest (third round of the Delphi process)

- Respond to revised questions (fourth round of the Delphi process)

Fig. 1. Delphi participants.

rope since 2005 for the treatment of moderate to severe plaque psoriasis in adults who failed to respond to, who have a contra-indication to or who are intolerant to other systemic therapies, including methotrexate (MTX).

Physicians using biological therapies have to make a number of decisions concerning patient management. These include: when to initiate biological agent therapy, determination of whether a patient should continue therapy after initial induction and issues about long-term patient management. Despite the broad use of infliximab beyond the formal clinical trial setting, there are no comprehensive guidelines for infliximab use in patients with psoriasis. This survey was therefore conducted with the objective of assessing current practices and generating pragmatic recommendations regarding the use of infliximab at key decision points in the management of patients with moderate to severe psoriasis:

- considerations at the start of biological therapy;

- considerations when continuing biological therapy beyond 12 weeks;
- considerations for long-term treatment with a biological agent;

- issues related to long-term patient management.

It is hoped that this collaborative effort will enhance the appropriate and safe use of infliximab in clinical practice and therefore contribute to improvements in management outcomes.

\section{Methods}

\section{Survey Participants}

Three dermatologists with extensive experience in using biological agents and involved in numerous clinical trials investigating biological therapy in plaque psoriasis constituted the steering group, outlining the topics for the survey and providing input on survey design and methodology (fig. 1). The survey was screened by these non-participating group members and tested by individuals outside the field of dermatology to evaluate readability and identify potential ambiguities in the questionnaire.

Eleven additional dermatologists from Europe and Canada agreed to participate in the modified Delphi survey developed and directed by the steering group. These physicians were selected based on their experience with using infliximab in the longterm treatment of patients with moderate to severe psoriasis in both clinical trials and clinical practice (fig. 1). This expert panel was made up of members who each treated a median of 100 chronic plaque psoriasis patients in an average month, nearly one third of whom were considered to have 'moderate to severe' disease and were therefore eligible for treatment with a biological agent, according to the current US labelling of these agents.

Delphi Methodology

The Delphi methodology, as developed by Linstone and Turoff [1], is an evidence-based approach characterised as 'a method for structuring a group communication process so that the process is effective in allowing a group of individuals, as a whole, to deal with a complex problem'. There are several subtypes of this Delphi system, with a number of key features in common: the recruitment of a group of expert participants, repeated distribution of a master questionnaire and anonymous collection of responses, analysis of results between rounds and the sharing of those results to allow individuals to re-evaluate and modify their answers as desired [1]. In general, this approach will yield a merging of opinions and serves to build consensus amongst the group.

A key strength of the Delphi methodology is that it can be used to explore different positions and the pros and cons of an argument, in some cases becoming a forum for the investigation helping to clarify ideas and opinions $[1,2]$. As the field has developed, a modification of the traditional Delphi methodology has been made to solve issues of how to reach a consensus [3]. This modification, termed the 'Collaborative Delphi', is part of a larger process in which both surveys and physical on-site meetings are used to establish a consensus [3]. This necessitates the participants' surrendering their anonymity during meetings, although individual responses remain confidential in order to preserve objectivity during survey completion. 
The consensus statements presented here have employed this Collaborative Delphi methodology via the administration of 2 rounds of an anonymous survey, followed by a meeting of the participants as the third round of the Delphi process. The expert panel reached consensus at the end of the on-site round on the topics of interest and developed 2 further questions concerning the long-term use of infliximab, which were then sent to the panel as round 4 of the Delphi process (fig. 1).

\section{Questionnaire Design}

Throughout the Delphi process, the panel was asked to fill in a questionnaire consisting of 3 main topic areas. In the first section, the participating physicians of the expert panel were asked questions pertaining to the use of biological agents at various stages of treatment: at initiation, during the normal course and for long-term therapy. The second section explored views on the aims of treatment and the use of measurement tools to assess disease, while the third section focused on the use of infliximab, in particular for the long-term treatment of psoriasis. The following aspects of the long-term treatment with infliximab were addressed:

- evaluation of treatment success after 6 and 12 months;

- cessation of infliximab treatment;

- reintroduction of infliximab following a period of withdrawal from therapy.

Questions were designed to assess areas of agreement, relevance, importance and likelihood of action. A Likert scale of 1-9 was used, where a score of $1,2,3$ or 4 indicated degrees of disagreement with the statement in question and a score of $6,7,8$ or 9 indicated increasing degrees of agreement. A score of 5 was considered neutral. The anonymity of individual answers was ensured by means of a numerical identification system known only to the administrators of the survey.

\section{Data Analysis}

Following the initial completion of the questionnaire by the expert panel, the responses were analysed on a group basis. On an ordinal scale such as the one used here, the sample mean would be unduly influenced by outlying values or extreme opinions. To provide a more appropriate measure of the main tendency, the sample median was used instead. The median scores were revealed to the participants in a second round of questioning. Following this input round, and prior to the assembly of the expert panel, the variability of responses was determined using the interquartile range, which is essentially a measure of the distance between the 75th and 25th percentile and highlights the middle $50 \%$ of the responses. The interquartile range is therefore not influenced by outliers or extreme responses and thus provides a stable measure of the range of responses.

Consistent with the Collaborative Delphi method, the third round of the process involved an on-site meeting of the expert panel. The results of the survey were presented by the steering group, and key issues pertaining to specific patient qualities and the cost, efficacy and safety of biological therapy were discussed in order to evaluate their relevance to the course of patient treatment. The median Likert score of each question was refined to clarify the degree of agreement with the statement or issue presented. Topics or statements with a score of 1 or 2 were regarded as highly irrelevant, highly unsuitable or highly unlikely; those with a score of 3 or 4 were considered irrelevant, unsuitable or unlikely; a score of 5 indicated neutrality; characteristics or items with a score of 6 or 7 were considered relevant, suitable or likely; and those scoring 8 or 9 were regarded as highly relevant, highly suitable or highly likely.

\section{Results}

\section{Recommendations during Therapy}

Whether initiating treatment or making decisions that will affect its course, it is essential to assess the severity of the patient's skin symptoms/signs and account for the presence of associated conditions such as PsA, pruritus, bleeding of psoriatic lesions and the involvement of nails or genital areas. Involvement of the nails can have a considerable effect on a patient's quality of life (QoL); in one report, over $50 \%$ of patients with psoriasis of the nails experienced pain related to nail changes and restrictions in their daily activities [4]. The degree of impairment of the patient's QoL is highly relevant at all stages of patient care. Symptoms of depression have been observed in patients with psoriasis and should also be assessed $[5,6]$.

The panel agreed that a general examination of the patient's health status, including vital signs and complementary tests, should be performed at follow-up visits. Particular emphasis should be placed on the current infection status, including tuberculosis, as well as the patient's current cardiac and/or hepatic function. Guidelines on patient selection and monitoring should be consulted when appropriate. As guidelines differ considerably among countries, it is beyond the scope of this paper to discuss them here. Physicians should refer to the specific guidelines for their own countries prior to initiating therapy.

\section{Initiation of Biological Treatment}

Because the cost of biological treatment is considerable and there is the potential for rare but serious side effects, it is essential to select the appropriate type of patient who will derive the greatest benefit from biological treatment (table 1). When first deciding on a course of treatment, the panel agreed that it is important to consider the initial level of efficacy achievable in the majority of patients, with the goal of achieving significant clinical improvement. The consensus of the panel was that an improvement in symptoms of at least $75 \%$ would be most meaningful to the patient.

The age of the patient and the experience in using biological therapies in similar patients should also be taken into account, along with the type of psoriasis being treat- 
Table 1. Key decision points when treating with biological agents

\begin{tabular}{|c|c|c|c|}
\hline \multirow[t]{2}{*}{ Assessment } & \multicolumn{3}{|l|}{ Treatment decision point } \\
\hline & initiation & continuing after 12 weeks & long term \\
\hline \multirow[t]{2}{*}{ Efficacy } & Initial level of efficacy achievable & $\begin{array}{l}\text { Results achieved (with respect to efficacy and } \\
\text { safety) }\end{array}$ & \\
\hline & Rapid clinical improvement & Likely level of sustained efficacy achievable & $\begin{array}{l}\text { Likely level of sustained efficacy achievable } \\
\text { Change in efficacy with long-term use }\end{array}$ \\
\hline \multirow{7}{*}{$\begin{array}{l}\text { Patient } \\
\text { factors }\end{array}$} & Age of patient & & Age of patient (health status changes likely \\
\hline & $\begin{array}{l}\text { Patient history of biological therapy } \\
\text { (including previously failed treatments) }\end{array}$ & & in elderly patients) \\
\hline & Severity of skin symptoms/signs & Severity of skin symptoms/signs & Severity of skin symptoms/signs \\
\hline & Patient QoL & Patient QoL & Patient QoL \\
\hline & $\begin{array}{l}\text { Special situations (PsA, involvement of } \\
\text { nails, face or genital area) }\end{array}$ & $\begin{array}{l}\text { Special situations (PsA, involvement of nails, } \\
\text { face or genital area) }\end{array}$ & $\begin{array}{l}\text { Special situations (PsA, involvement of } \\
\text { nails, face or genital area) }\end{array}$ \\
\hline & $\begin{array}{l}\text { Patient health status (infections, impaired } \\
\text { cardiac and/or hepatic function) }\end{array}$ & $\begin{array}{l}\text { Patient health status (infections, impaired } \\
\text { cardiac and/or hepatic function) }\end{array}$ & $\begin{array}{l}\text { Patient health status (infections, impaired } \\
\text { cardiac and/or hepatic function) }\end{array}$ \\
\hline & History of malignancy & $\begin{array}{l}\text { Comorbidities [metabolic disorders, } \\
\text { inflammatory disorders, cardiovascular } \\
\text { disease (NYHA class III/IV)] }\end{array}$ & $\begin{array}{l}\text { Comorbidities [metabolic disorders, } \\
\text { inflammatory disorders, cardiovascular } \\
\text { disease (NYHA class III/IV)] }\end{array}$ \\
\hline \multirow[t]{2}{*}{ Safety } & General safety & General safety & General safety \\
\hline & Potential for adverse events & Potential for adverse events & Potential for adverse events \\
\hline Cost & & & Reimbursement status \\
\hline
\end{tabular}

ed and the patient's previous treatment history, including those that they may have failed.

Patients considered suitable or highly suitable for infliximab therapy (according to the median Likert scores determined by the expert panel) are those who have:

- moderate to severe psoriasis and failed 2 or more systemic therapies;

- PsA in association with skin symptoms;

- associated psoriasis of the nails, face or genitals;

- moderate to severe psoriasis in conjunction with $\mathrm{CD}$ or a concomitant metabolic syndrome;

- a Psoriasis Area and Severity Index (PASI) $\geq 20$;

- improvement of $<50 \%$ (PASI 50) with a previous nonbiological or alternate biological therapy;

- a score of $>2$ on the Physicians' Global Assessment (PGA) or a reduction of less than 5 points on the Dermatology Life Quality Index (DLQI) while on a previous non-biological or alternative biological therapy.

The patient's history of skin cancer or other malignancies should be evaluated to determine their impact on a potential therapy. A patient who has a history of or currently has melanoma (not melanoma in situ) should be excluded from infliximab therapy; a diagnosis of nonmelanoma skin cancer within the same time frame was not considered a limiting factor to treatment. Patients with other non-dermatological underlying conditions

Long-Term Treatment of Psoriasis with Infliximab should be evaluated on an individual basis to determine whether biological therapy would be appropriate.

\section{Continuing Treatment after Induction Dosing}

In considering continuation of treatment after 12 weeks (or 3 infusions) of biological therapy, it is important to evaluate the level of sustained efficacy achievable in the majority of patients. After 12 weeks of biological therapy, treatment should be continued as long as the patient has achieved at least PASI 50. If the patient has not achieved PASI 50, the current treatment may be optimised by several methods. These may include decreasing the interval between infusions (e.g. from every 8 weeks to every 6 weeks), increasing the dose of drug administered and/or introducing a supplementary therapy such as a topical treatment or MTX. Data from well-controlled clinical trials would need to be obtained in order to determine the optimal course of action.

Patients should be screened for the presence of comorbidities related to chronic inflammation (e.g. obesity, diabetes and heart disease) and other chronic inflammatory conditions such as CD and PsA. Treatment should be discontinued in any patient who experiences severe hypersensitivity or a life-threatening allergic reaction. It is essential to closely observe patients for any other adverse events and to monitor patient safety in general. 
Fig. 2. Algorithm for the initial course of treatment of moderate to severe psoriasis with infliximab.

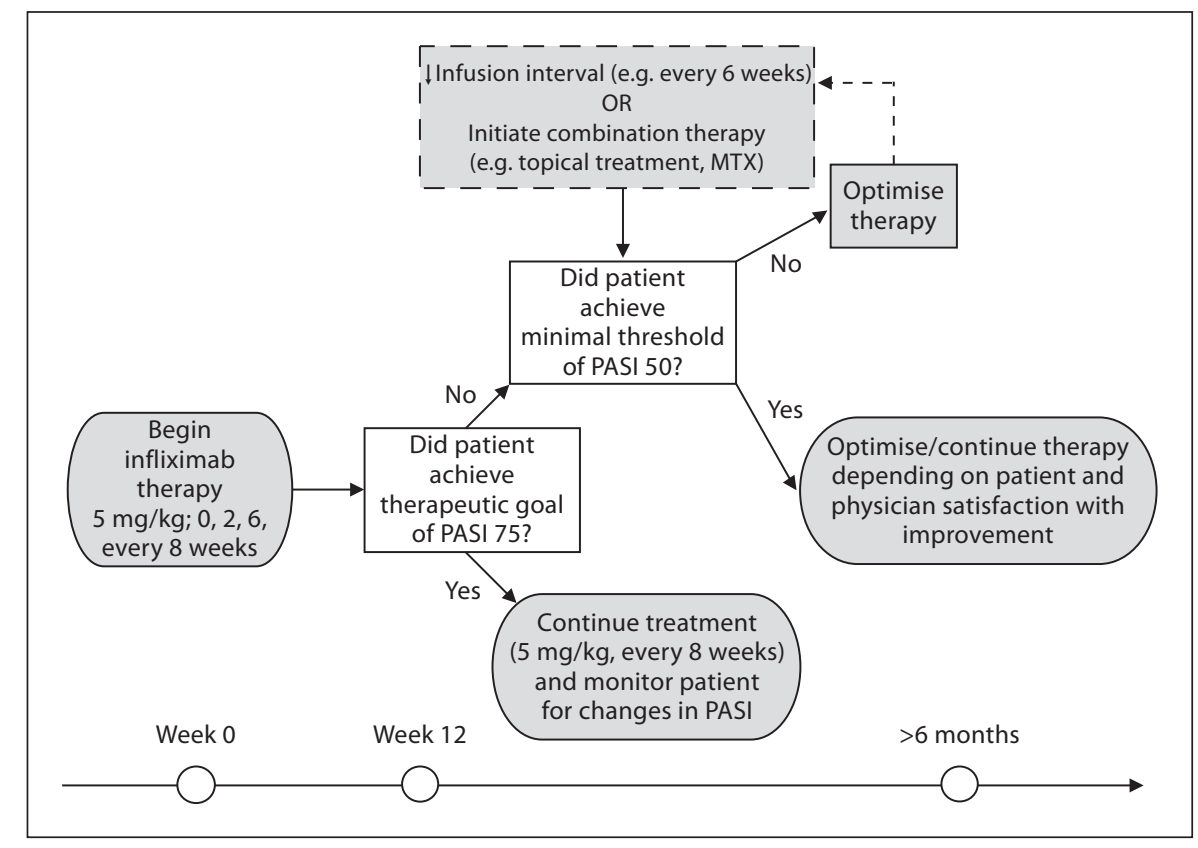

Long-Term Treatment with Biological Therapy

The panel agreed that the level of sustained efficacy achievable is highly relevant to the decision to continue long-term biological therapy. Patients should be monitored for periods of suboptimal response to biological therapy with long-term use. Monitoring for general safety concerns and comorbidities should continue, particularly in older patients, since changes in health status are common.

It may be required in some cases that a patient meets certain clinical criteria in order to be considered for longterm ( $>6$ months) therapy. As long as the patient achieves at least PASI 50, treatment should not be stopped. If the patient fails to achieve PASI 50 after 6 months of infliximab use in spite of efforts to optimise therapy, this may be considered a 'non-response' and it may be appropriate to consider an alternate treatment regimen. In patients who have been on infliximab for at least 1 year, the threshold for a positive response is less clear; a patient who may already have achieved PASI 75 after several weeks of treatment may or may not be satisfied with that level of skin clearance. The panel recommends that after 1 year of infliximab the continued course of action be determined based on the specific goals and needs of the individual patient.

Following 6-12 months of successful treatment with infliximab, its dose should not be decreased and the infusion interval should not be extended. However, if a pa- tient has initially responded very well to infliximab $(\geq$ PASI 75 ) for the first 12 weeks but experiences a loss of response ( $<$ PASI 50) at 6 months, it would be appropriate to reduce the interval between infusions to optimise therapy. It may also be beneficial to introduce a topical treatment in addition to biological therapy or use infliximab in combination with MTX. Data are insufficient to make a recommendation on the point at which switching to an alternative biological therapy, or which biological agent, is prudent. This general treatment algorithm is summarised in figure 2.

\section{Recommendations on Treatment Goals}

Evaluation of Efficacy on Skin Symptoms

Based on the panel's experience both in clinical trials and regular practice settings, PASI 75 should be the criterion for successful treatment, as this represents a meaningful improvement to the patient. PASI 50 is the lowest level of improvement acceptable after 12 weeks of biological therapy. If PASI 50 is not obtained at that time, the panel agreed that it would be appropriate to consider switching treatment to an alternative biological agent.

The PGA, though not as widely used as the PASI, is an appropriate tool for the measurement of psoriasis severity. When using the PGA, the panel thought that the goal of biological therapy should be to achieve a score of 2 (mild psoriasis) or better. The patient should achieve at least a PGA score of 3 (moderate psoriasis) after 12 weeks 
Table 2. Acceptable threshold of improvement with biological therapy

\begin{tabular}{|c|c|c|}
\hline $\begin{array}{l}\text { Measure- } \\
\text { ment } \\
\text { tool }\end{array}$ & $\begin{array}{l}\text { Level of improvement } \\
\text { at which treatment is } \\
\text { considered successful }\end{array}$ & $\begin{array}{l}\text { Level of improvement } \\
\text { below which treatment } \\
\text { should be discontinued }\end{array}$ \\
\hline PASI & PASI 75 & PASI 50 \\
\hline PGA & $\begin{array}{l}\text { Score of } 2 \text { : } \\
\text { 'mild' symptoms }\end{array}$ & $\begin{array}{l}\text { Score of 3: } \\
\text { 'moderate' symptoms }\end{array}$ \\
\hline DLQI & Mean decrease of $\geq 5$ points & Mean decrease of $<5$ poin \\
\hline
\end{tabular}

Table 3. Discontinuation/reintroduction of infliximab therapy following change in the patient's health status

\begin{tabular}{ll}
\hline Reason for discontinuation of infliximab therapy & $\begin{array}{l}\text { Reintroduction of } \\
\text { infliximab following } \\
\text { return to normal } \\
\text { health status }\end{array}$ \\
\cline { 2 - 2 } & $\begin{array}{l}\text { highly some- un- } \\
\text { likely what likely } \\
\text { likely }\end{array}$ \\
\hline
\end{tabular}

Requires surgery for an unrelated event Requires vaccination for vacation/travel Becomes pregnant while under therapy

Experiences non-melanoma skin cancer

Experiences TB infection

Experiences clinically significant liver dysfunction (ALT $\geq 5$ times normal)

Experiences a serious bacterial/fungal infection

Experiences a severe infusion reaction

Experiences another type of serious adverse event

Experiences a severe hypersensitivity reaction

Experiences melanoma

Experiences lymphoma/other malignancy

\begin{tabular}{lll}
$\checkmark$ & & \\
$\checkmark$ & & \\
$\checkmark$ & & \\
$\checkmark$ & & \\
& $\checkmark$ & \\
& & \\
& $\checkmark$ & \\
& $\checkmark$ & \\
& $\checkmark$ & \\
& $\checkmark$ & \\
& & $\checkmark$ \\
& & $\checkmark$ \\
& & $\checkmark$ \\
\hline
\end{tabular}

$\mathrm{TB}=$ Tuberculosis; $\mathrm{ALT}=$ alanine aminotransferase.

of treatment; otherwise, discontinuation should be considered.

These recommendations regarding the acceptable threshold of improvement following biological therapy in adults with psoriasis are summarised in table 2.

Evaluation of Improvements in Patient QoL

Successful management of psoriasis encompasses the assessment of a patient's QoL in addition to the evaluation of physical symptoms. Symptoms of depression in patients with psoriasis have been shown to be related to impairments in health-related QoL arising from dissatisfaction with treatment and illness-related stress [6]. The DLQI complements the existing tools for the evaluation of symptom severity and is an important measure of the patient's QoL. Although the DLQI is by nature patient specific, the expert panel determined that an average decrease of 10 points would represent a meaningful improvement to a patient and should be the standard goal for biological treatment (table 2). Further, they agreed that a reduction in the DLQI of less than 5 points would indicate the need to pursue other treatment options. The individual nature of each patient's disease should be taken into account to determine whether the treatment is having a meaningful effect on QoL.

\section{Additional Areas of Patient Management}

It may be necessary in some cases to discontinue infliximab therapy due to a temporary change in the patient's health. Once the patient's general health returns to normal, treatment can then be resumed. Reintroduction of infliximab after a short break in treatment (i.e. approximately 8 weeks) should not compromise safety and efficacy. The majority of patients will regain their response following the reintroduction of infliximab after a treatment-free interval of more than 8 weeks; in instances where this does not occur, combination therapy (e.g. infliximab given in conjunction with MTX) may be appropriate.

Discontinuation/Reintroduction of Infliximab due

to Change in Patient Health Status

Patients who become pregnant while being treated with infliximab, who are scheduled for surgery for a related or an unrelated condition, or who require certain vaccinations should suspend infliximab therapy temporarily. It is highly likely that infliximab treatment would then be resumed once the patient's health has returned to normal. Infliximab therapy should also be discontinued in any patient with a current diagnosis of clinically relevant liver dysfunction (alanine aminotransferase $\geq 5$ times the upper limit of normal), tuberculosis, bacterial sepsis, or invasive fungal or other opportunistic infection. Once the infection has cleared, infliximab may be reintroduced.

Some patients may experience an infusion reaction; many of these are mild and can be managed by slowing the rate of infusion. However, in patients who experience a severe infusion reaction or some other type of serious adverse event despite slowing the infusion rate, infliximab treatment should be discontinued temporarily; if the patient experiences a severe hypersensitivity reaction, it may not be advisable to resume therapy. Infliximab should also be stopped due to a diagnosis of melanoma, 
Fig. 3. Rationale for reintroduction of infliximab therapy after discontinuation for reasons other than safety.

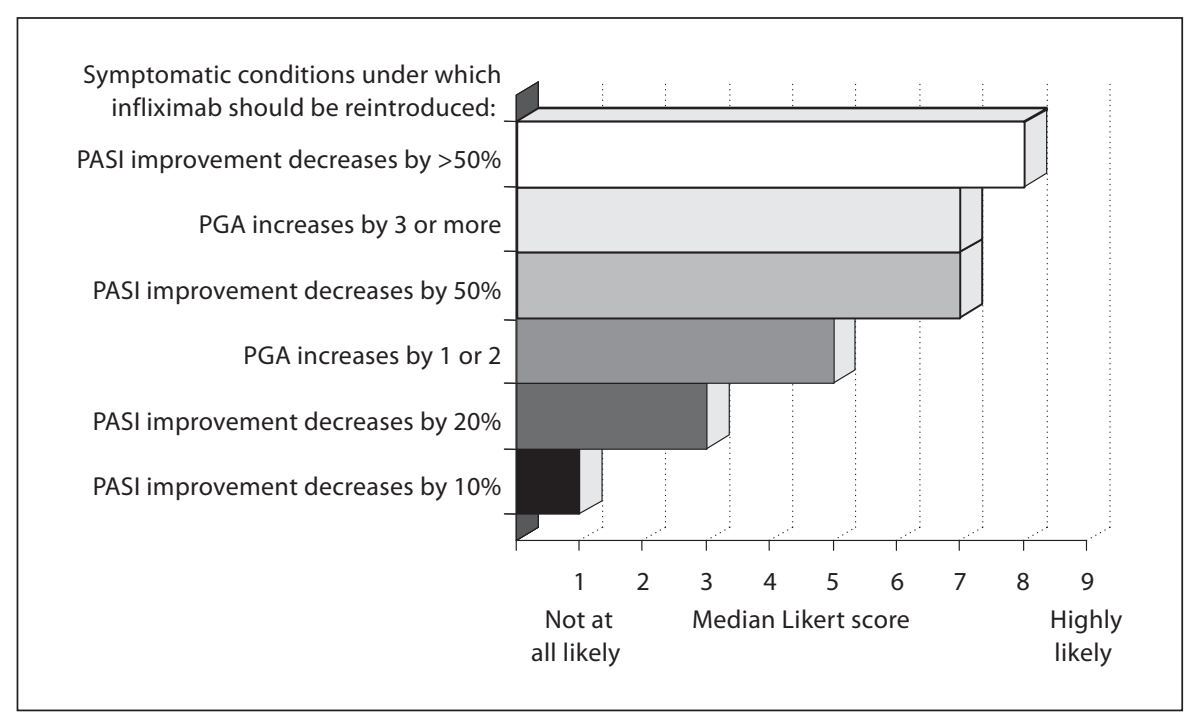

non-melanoma skin cancer, lymphoma or another type of malignancy. Although the patient may resume treatment following surgical removal of non-melanoma skin cancer, reintroduction of infliximab following other malignancies is not recommended. A summary of these potential situations that may require the discontinuation of infliximab treatment and the likelihood of reintroduction is presented in table 3.

Rationale for Reintroduction of Infliximab after Discontinuation due to Reasons Other than Safety

If the patient's PASI improvement decreases by more than $50 \%$ or the PGA score increases by 3 or more points, a change in therapy may be warranted. However, a patient who exhibits a decrease in PASI improvement by $10-20 \%$ or an increase in PGA of only 1 or 2 points may be considered as being treated successfully if the absolute level of skin clearance and improvement in QoL remains acceptable; such a patient should remain on infliximab.

If infliximab has been discontinued, the patient should be monitored carefully to determine the optimal conditions under which infliximab may be reintroduced. The same guidelines described above - a decrease in PASI improvement by $50 \%$ or more or an increase in PGA of 3 or more points - should be applied when deciding whether to resume infliximab treatment. The likelihood that infliximab is reintroduced to a treatment-free patient following a measurable worsening of symptom severity is summarised in figure 3 . It should be noted that the decision to reintroduce infliximab following a treatment-free interval is very patient specific; the degree of improve- ment, or lack thereof, should be evaluated on an individual basis to assess whether there is a significant effect on the patient's QoL before making any changes in therapy.

\section{Conclusion}

As more physicians start their patients on biological therapy, it is important to provide guidance with regard to patient management over the long term of this chronic disease. Current treatment guidelines do not provide this information on long-term management, and until they are updated it is critical to ensure that some data on best practice be available. The questionnaire used in this Delphi survey was developed by an expert steering group and focused on questions and situations that had been encountered during their experience in treating patients with infliximab.

The expert panel subsequently reached a consensus in several areas including the need to modify the expected goal of treatment with biological agents. As it is now quite reasonable to expect a patient to safely achieve at least PASI 75, one should not compromise on attaining this level of success. QoL is strongly influenced by improvement of skin symptoms, and although QoL improvement can be patient specific, the panel agreed that an average decrease of 10 points on the DLQI scale, which would represent a meaningful improvement to the patient, should be considered the standard goal for treatment with biological agents. 
This consensus statement clarifies key decision points on how to manage patients with moderate to severe psoriasis in the long term and provides guidance on the longterm management of those receiving infliximab. The results discussed here provide a large basis of experienced opinions for the long-term use of infliximab in treating moderate to severe plaque psoriasis. It is accepted that many of the principles outlined here are based on opinion due to the lack of clinical data in some areas, and this is an acknowledged limitation of this type of approach. Robust, data-based investigation into the long-term clinical use of infliximab is needed to strengthen and enhance these expert-based recommendations. Ideally the combination of robust data and expert experience and opinion will contribute to the appropriate and safe management of patients in the long term.

\section{Acknowledgement}

The consensus meeting was financially supported by Schering-Plough. The sponsor did not participate in the expert panel discussion.

\section{Conflict of Interest Statements}

Esteban Daudén has participated in scientific advisory boards for Abbott, Wyeth, Schering-Plough and LEO Pharma. Robert Gniadecki has been an investigator for and received research grants from Schering-Plough, Abbott, Wyeth and Merck Serono. Richard Langley has been an investigator and served on scientific advisory boards for Schering-Plough, Wyeth, Amgen, Biogen Idec, Serono, Genentech, Abbott, Centocor and Ortho Biotech. Ulrich Mrowietz has received honoraria for lectures and participation in advisory boards from Abbott, Biogen Idec, Centocor, Jansen-Cilag, LEO Pharma, Merck Serono, Essex Pharma/Schering-Plough and Wyeth. Alexandra Ogilvie has been an investigator and served on scientific advisory boards for Centocor, Schering-Plough, Essex Pharma (national), Wyeth, Abbott and Serono. Jean-Paul Ortonne has been an investigator, speaker and advisor for Schering-Plough, Abbott, Merck Serono, Centocor, Wyeth, Janssen-Cilag, Meda Pharma, Pierre-Fabre and Galderma. He has also been an investigator and speaker for LEO Pharma. Norbert Reider has been an investigator in clinical studies for Schering-Plough, Abbott, Serono and Wyeth and has been a speaker in symposia sponsored by Schering-Plough and Wyeth. Kristian Reich has been a consultant and/or paid speaker for Abbott, Biogen Idec, Centocor, Essex Pharma, Schering-Plough, Serono and Wyeth. Jean-Hilaire Saurat has received honoraria for serving on advisory boards for Centocor, Abbott, Wyeth and Serono.

\section{References}

1 Linstone HA, Turoff M (eds): The Delphi Method: Techniques and Applications. Reading, Addison-Wesley, 1975, pp 291321.

2 Rauch W: The Decision Delphi. Technol Forecast Soc Change 1979;15:159-169.
3 Rowe H: 'The 'Collaborative' Delphi' symposium. Annual Sustainable Rangelands Roundtable Meeting, Phoenix, 2005.

4 de Jong EM, Seegers BA, Gulinck MK, Bozeman JB, van de Kerkhof PC: Psoriasis of the nails associated with disability in a large number of patients: results of a recent interview with 1,728 patients. Dermatology 1996; 193:300-303.
5 Esposito M, Saraceno R, Giunta A, Maccarone $\mathrm{M}$, Chimenti S: An Italian study on psoriasis and depression. Dermatology 2006; 212:123-127.

6 Schmitt JM, Ford DE: Role of depression in quality of life for patients with psoriasis. Dermatology 2007;215:17-27. 